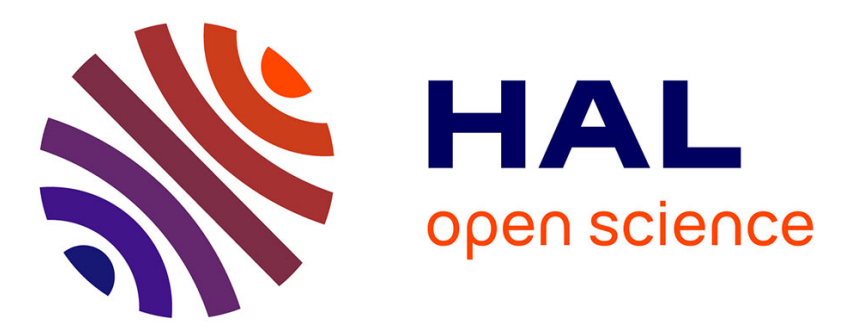

\title{
NMR and XRD quantification of bound and free water interaction of spruce wood fiber
}

Chady El Hachem, Kamilia Abahri, Sébastien Leclerc, Rachid Bennacer

\section{To cite this version:}

Chady El Hachem, Kamilia Abahri, Sébastien Leclerc, Rachid Bennacer. NMR and XRD quantification of bound and free water interaction of spruce wood fiber. Construction and Building Materials, 2020, 260, pp.120470. 10.1016/j.conbuildmat.2020.120470 . hal-02928311

\section{HAL Id: hal-02928311 \\ https://hal.univ-lorraine.fr/hal-02928311}

Submitted on 2 Sep 2020

HAL is a multi-disciplinary open access archive for the deposit and dissemination of scientific research documents, whether they are published or not. The documents may come from teaching and research institutions in France or abroad, or from public or private research centers.
L'archive ouverte pluridisciplinaire $\mathbf{H A L}$, est destinée au dépôt et à la diffusion de documents scientifiques de niveau recherche, publiés ou non, émanant des établissements d'enseignement et de recherche français ou étrangers, des laboratoires publics ou privés. 
NMR and XRD quantification of bound and free water interaction of spruce wood fiber

\title{
Chady EI Hachem ${ }^{1}$, Kamilia Abahri ${ }^{1}$, Sebastien Leclerc ${ }^{2}$, Rachid Bennacer ${ }^{* 1,3}$
}

Université Paris-Saclay, ENS Paris-Saclay, CNRS, LMT - Laboratoire de Mécanique et Technologie, 94235, Cachan, France.

${ }^{2}$ LEMTA, University of Lorraine \& CNRS, France

${ }^{3}$ ECAM-EPMI, Bd de 1'Hautil, 95000 Cergy, France

* Corresponding author:

E-mail address: rachid.bennacer@ens-paris-saclay.fr

\begin{abstract}
The quantification of the bound and free water within spruce wood and the water and solid matrix interactions are still unknown by the researchers.

Many specimens were firstly studied by Nuclear Magnetic Resonance function of the relative humidity. For each test, the bound and free water were calculated. After that, spruce wood specimens were studied by X-Ray Diffraction, function of the relative humidity. For each test, the crystalline and amorphous phases were quantified.
\end{abstract}

This paper presents innovative results concerning the bound and free water uptake within wooden materials, and the water and wooden solid matrix interactions.

\section{Keywords}

Bound water, free water, fiber saturation point, swelling, spruce wood, Nuclear Magnetic Resonance, X-Ray Diffraction

\section{Introduction}

Because of its thermal insulation properties and energetic performances, wood is being widely used in the construction field (Dodoo et al., 2012). Spruce wood, the studied material in this paper, is particularly known as well for its mechanical, thermal and hygric properties.

This material presents an anisotropic microstructure, characterized by three principle directions: longitudinal, radial and tangential. However, this anisotropy is accompanied by a multi-scale behavior. In fact, at the microscopic scale, spruce wood is constituted of fibers (tracheids and parenchyma). Each fiber is formed of cell walls, containing porosity holes 
(Mäkinen et al., 2007; Rafsanjani et al., 2012). This multi-scale behavior is as well observed at the cell walls' chemical constituents of wood, and biomaterials in general (Salvati et., 2018). In fact, the cell walls are constituted of many layers, in which mainly cellulose (crystalline and amorphous), hemicellulose and lignin coexist. Each of these constituents is characterized by its own thermal, hygric and mechanical properties, which strongly affect the macroscopic behavior of spruce wood (Derome et al., 2013; Almeida et al., 2014; Cueff et al., 2018).

On the other hand, wood is a hygroscopic bio-based material, often in interaction with humidity (Gezici-Koç et al., 2017; Zlahtic-Zupanc et al., 2018; Zhou et al., 2019). Therefore, it tends to swell or shrink, whether its water content increases or decreases respectively (Bonnet et al., 2017; El Hachem et al., 2017; El Hachem et al., 2019). In fact, the cell walls dimensional variations take place following the absorption (or desorption) of bound water, whereas free water does not affect the wood structure, because it occupies the space within the cavities (porosity). Along the sorption cycle, the material absorbs first the bound water until the cell walls get fully saturated (Gezici-Koç et al., 2017). This state of the material corresponds to the Fiber Saturation Point (FSP) (Eitelberger et al., 2011). Once the FSP is achieved, there is no more swelling within the material, and every additional increase in the water content of the material is assimilated to free water within the porosity (Gezici-Koç et al., 2017).

The microscopic dimensional variations caused by the relative humidity changes have been previously revealed (Derome et al., 2013; Almeida et al., 2014; Guo et al., 2015; El Hachem et al. 2017; El Hachem et al., 2018). In fact, at the cell walls' scale, there is an instability related to the swelling and shrinkage caused by the relative humidity variations, and particularly to the various chemical components properties (Wang et al., 2008; Neagu et al., 2007; Guo et al., 2015). All these factors strongly influence the cell walls' response when subjected to various environmental conditions of relative humidity and temperature.

There is still lack of knowledge in literature concerning the interaction of the wood cell with relative humidity. There is no real distinction between the free and bound water with precise estimation when the FSP occurs.

In a previous work (El Hachem et al. 2017), we investigated by X-ray tomography the pore size and the fibers dimensions' evolution function of the water content of wood. However, 
the X-ray tomography technique is limited to distinguish the water interaction within the wood microstructure.

Recently, Nuclear Magnetic Resonance (NMR) technique was used for wooden material (Almeida et al., 2008; Hernández and Cáceres, 2010; Fourmentin et al., 2016; Gezici-Koç et al., 2018; Zhou et al., 2018; Rostom et al., 2019). For example, Almeida et al. (2008) studied the water restitution of initially saturated Douglas wood when subjected to dried conditions. On the other hand, Fourmentin et al. (2016) studied the water adsorption/uptake of initially dried specimens of hemp when subjected to in-situ saturated conditions. The principal results of such a technique is the water content quantification.

Therefore, the present work aims to study the coupling between the hygric response of bound and free water at the microscopic scale and the local swelling related to the chemical components of the material, with respect to the FSP. In addition, the material in question is spruce wood, being a sapwood having a density of $450 \mathrm{Kg} / \mathrm{m}^{3}$, which has not been studied yet which such techniques (NMR and XRD).

The first part of the paper concerns the bound and free water quantification along the sorption cycle, using NMR. A special post-processing procedure and a specific device have been developed for this purpose. The relative humidity interactions with the material, at the scale of the cell walls' chemical constituents will be afterwards treated by X-Ray Diffraction (XRD) methods. Once more, a specific procedure has been set up in order to do the experiment considering each humidity state of the material. Finally, a global interpretation of the results will take place, in order to highlight the physical interactions at different scales.

\section{Material and methods}

An accurate study of bound and free water interactions with the solid matrix of wood requires very well organized and refined experiments' protocols. Both adopted techniques, Nuclear Magnetic Resonance (NMR) and X-ray Diffraction (XRD), as well as the corresponding experimental protocols and the specific devices used to maintain a constant relative humidity in each experiment will be first discussed.

\subsection{Nuclear Magnetic Resonance (NMR)}

NMR is a non-destructive experimental technique used in the characterization of materials. It consists in modifying the magnetization of nuclear spins of certain atoms, due to the effect of 
two magnetic fields $\mathrm{B}_{0}$ and $\mathrm{B}_{1}$. The magnetic field $\mathrm{B}_{0}$ presents a high and fixed intensity, whereas the magnetic field $B_{1}$ is rotational (Keeler, 2011). The hydrogen atom is the most sensible nucleus (Arends et al., 2018; Wang et al., 2018) due to its $1 / 2$ spin and high intrinsic gyromagnetic moment of $42.6 \mathrm{MHz} / \mathrm{T}$. In addition, this atom is very abundant, which leads to a well-defined resonance phenomenon.

This technique is principally used in the medical field, and for organic molecules characterization purposes. Relaxometry methods were developed more recently for civil engineering applications. For example, this technique can be used to characterize the porous structure of materials, as well as the quantification of free water in the porosity and other water states that interact with the solid matrix of the material. In this work, only free water in the porosity, and bound water absorbed by the cell walls are identified. However, the signal coming from the chemical constituents of the wood is not detected with the experimental conditions used in this study.

An NMR experiment firstly consists in placing the sample studied in the homogeneous magnetic field $\mathrm{B}_{0}$. This is the basis of a macroscopic longitudinal magnetization $\mathrm{M}$ parallel to the intense field $\mathrm{B}_{0}$. Then, a radio frequency of short duration (a few microseconds), characterized by a rotating magnetic component $\mathrm{B}_{1}$, perpendicular to $\mathrm{B}_{0}$, causes the tilting of this magnetization on another plane. The longitudinal component of the induced magnetization will decrease, and the spins will be phased during the excitation of the atoms. This causes the transverse magnetization, which is the transverse component of the induced magnetization. At the end of the pulse, the nuclei being in an unfavorable energy level, magnetization will tend to return to its initial position. This is known as the relaxation phenomenon. An example showing the relaxation effects following a $90^{\circ}$ pulse is shown in Figure 1.

There are two main types of relaxation, the transversal and the longitudinal relaxation. Evolution of the magnetization $\mathrm{M}$ with relaxation and magnetic field $\mathrm{B}$ is given by the Bloch equations 1 and 2 :

$\frac{\mathrm{dM}_{\mathrm{x}, \mathrm{y}}}{\mathrm{dt}}=\gamma(\overrightarrow{\mathrm{M}} \wedge \overrightarrow{\mathrm{B}})_{\mathrm{x}, \mathrm{y}}-\frac{\mathrm{M}_{\mathrm{x}, \mathrm{y}}}{\mathrm{T}_{2}}$ 
$\frac{\mathrm{dM}_{\mathrm{z}}}{\mathrm{dt}}=\gamma(\overrightarrow{\mathrm{M}} \wedge \overrightarrow{\mathrm{B}})_{\mathrm{z}}-\frac{\mathrm{M}_{0}-\mathrm{M}_{\mathrm{z}}}{\mathrm{T}_{1}}$

With $\mathrm{T}_{1}$ and $\mathrm{T}_{2}$ the time constants corresponding respectively to the longitudinal and transverse relaxation.

Brownstein and Tarr (1979) have proposed a theory that relates relaxation times to pore diameters. Therefore, each relaxation time measured in NMR corresponds to a given pore size. As a result, free water, located in the porosity, is easily discerned from the bound water that integrates into the cell walls.

\subsection{X-ray Diffraction (XRD)}

$\mathrm{XRD}$ is a technique that allows characterization of the crystalline structures of materials. A crystalline body is considered as an assembly of atomic planes more or less dense, called reticular planes. The characteristic distances separating these different planes are called interreticular distances. The existence of reticular planes and inter-reticular distances is at the basis of XRD.

When irradiating parallel beams of monochromatic X-rays, the atomic planes that form a crystalline material behave as a three-dimensional network. The X-ray beam is thus diffracted according to specific angles. This can provide information about the texture and degree of crystallinity of the material (Liang et al., 1997).

The theory of XRD was developed by W. L. Bragg. Its principle as well as its use are detailed in Bragg's relationship. Indeed, for an incident ray of monochromatic X-rays of wavelength $\lambda$, a ray is diffracted on Miller's index planes (h, k, l) if it satisfies Bragg's law (Langford and Louër, 1996). The wavelength $\lambda$ is between $0.1 \AA$ for hard X-rays, and $50 \AA$ for soft X-rays. The equation governing the Bragg relation is given in equation 3. This equation links the Bragg angles $\theta$, the wavelength $\lambda$, the inter-reticular distance of the family of planes $\{h, k, 1\}$ which diffract the incident rays $\mathrm{d}_{\mathrm{hkl}}$ and the diffraction order $\mathrm{n}$.

$\sin \theta=\frac{\mathrm{n} \lambda}{2 \mathrm{~d}_{\mathrm{hkl}}}$

The principle of Bragg's law is illustrated in Figure 2. 
The characterization of crystalline materials' structures is made from the diffraction laws. In fact, two diffraction modes exist: when the source is monochromatic, the incident X-ray beam has X-rays of the same wavelength, whereas if the source is polychromatic, the incident $\mathrm{X}$-ray beam has X-rays of different wavelengths.

The X-rays diffracted during the angular sweep of the irradiated surface of the sample are collected by an X-ray detector. The recorded signal provides the diffraction diagram, and represents the intensity measured by the detector as a function of the value of $2 \theta$. From the angles that correspond to the observed diffraction peaks, the inter-reticular distances of the families of crystalline planes can be calculated, using Bragg's law. This is the way the crystallographic structure of the material is identified.

At the cell walls' scale of wood, there are various chemical constituents. Hemicellulose and lignin are present only in the amorphous phase, whereas cellulose exists in the wood in crystalline and amorphous form. As a result, the crystalline peaks that will be detected in XRD should correspond to the crystalline cellulose. The chemical formula of the crystalline cellulose is $\left(\mathrm{C}_{6} \mathrm{H}_{10} \mathrm{O}_{5}\right)_{\mathrm{n}}$, and the corresponding chemical structure is shown in Figure 3.

The crystalline cellulose present in the cell walls, is of type $I_{\beta}$ and of monoclinic form, with the mesh characteristic parameters represented in table 1.

The XRD spectrum of crystalline cellulose, as well as the different crystalline planes are shown in Figure 4-a, based on the PDF-2 sheets (Powder Diffraction File 2), provided by the ICDD (International Centre for Diffraction Data). The diffraction graph shows two obvious peaks for $2 \theta$ values of $17.2^{\circ}$ and $26.4^{\circ}$. For the interpretations in sections 3.2 and 3.3, we will refer to the $2 \theta$ main peak of $26.4^{\circ}$, corresponding to plane [002].

Furthermore, some studies have been completed in the literature concerning the crystalline form of cellulose. For example, some values of inter-reticular distances and cellulose crystallinity, were calculated by Ju et al. (Ju et al., 2015):

$$
\begin{array}{ll}
- & \mathrm{d}_{002}=0.389 \\
- & \mathrm{d}_{10 \mathrm{i}}=0.531 \\
- & \mathrm{d}_{101}=0.593
\end{array}
$$


The geometric shape of the cellulose, as well as the main plane [002], and the inter-reticular distances d002 are shown in Figure 4-b-c.

\subsection{Experimental protocol for bound and free water quantification using NMR}

The originality of this study is to quantify the bound and free water within the wooden material, according to the FSP. To do so, a dry state (conditioning with silica gel), and relative humidity states of $78 \%$ and $95 \%$ were chosen for this study. This choice was made since the FSP is reached for a relative humidity greater than $78 \%$, and smaller than $95 \%$ (El Hachem et al., 2017). In addition, these two levels are identical to those adopted in the X-ray micro-tomography experiment, elaborated in a previous paper (El Hachem et al., 2017). That way, the results' interpretations will be more complementary.

Six spruce wood samples of dimensions $2.5 \times 2.5 \times 20 \mathrm{~mm}^{3}$ were prepared. Each sample was big enough to contain a complete reproduction cycle of wood, where early wood and latewood coexist. The $20 \mathrm{~mm}$ side of each sample was along the longitudinal direction of wood. All samples were first dried in dessicators using silica gel. Actually, the signal measured in NMR is proportional to the amount of material present in the tube. Therefore, each sample was placed in an NMR spectroscopy tube. In this way, as each sample has six sides exposed to the same environmental conditions, the mass balance of all samples will be achieved much faster than when only one equivalent large volume sample is used.

The NMR device used is a Spectroscopy-Bruker Minispec MQ20 ND-Series, with a ${ }^{1} \mathrm{H}$ proton resonance frequency of $20 \mathrm{MHz}$, at CRM2 (Laboratoire de Cristallographie, Résonance Magnétique et Modélisations) in Nancy, France. It is shown in Figure 5-a. The diameter of the NMR tube is $10 \mathrm{~mm}$. At each hygric state, a saline solution that guarantees a well-controlled relative humidity was placed in a small tube of $6 \mathrm{~mm}$ diameter, placed on a teflon support, all inside the NMR tube. This support has several holes in order to let moisture into the tube, and thus provide an environment with homogeneous humidity. The NMR tube was then closed and covered with a parrafin film to ensure that no moisture exchange will take place with the external environment. The assembly formed of the teflon support, the NMR tube, the tube containing the saline solution, and the sample is shown in Figure 5-b. 
As soon as the tube is put in the NMR spectroscopy device, the transverse relaxation time $\mathrm{T}_{2}$ is measured. Then the longitudinal relaxation time $T_{1}$ is evaluated every hour. Finally, when the mass balance is reached, the transverse relaxation time $T_{2}$ is measured once again.

$\mathrm{T}_{1}$ relaxation times were calculated using an inversion recovery procedure. 16 scans were performed per measurement point, with a $2 \mathrm{~s}$ recycle time, a gain of $90 \mathrm{~dB}$, and 50 points logarithmically spaced by an echo time between $0.1 \mathrm{~ms}$ and $1 \mathrm{~s} . \mathrm{T}_{2}$ relaxation times were measured using a Carr Purcell Meiboom Gill (CPMG) procedure. 512 scans were performed, with a recycle time of $2 \mathrm{~s}$, a gain of $90 \mathrm{~dB}$, an echo time of $80 \mu \mathrm{s}$, and 500 consecutive echoes acquired.

At each relative humidity level, once the mass balance is reached, the small tube containing the saline solution is extracted from the NMR tube, and is replaced by another tube of the same size containing another saline solution. Whenever the salt solution is changed, the procedure described for calculating the longitudinal and transverse relaxation times between two specific relative humidity conditions is completed.

The post-processing of the longitudinal and transverse relaxation times is done afterwards by Laplace inversion, using UPENWIN software (Borgia et al., 2000). The corresponding diagrams represent the amount of signal measured for each relaxation time. On the other hand, treatment with inverse Laplace transformation leads to fairly wide peaks. Each peak is representative of a pore size in which the hydrogen protons are located. As a result, the bound water located in the cell walls, and the free water located in the porosity can be identified, for each relative humidity level. On the other hand, for the spectroscopy, it is necessary to take into account the area under the peaks, and not the exact positioning of the peaks. This is shown in Figure 6-a. In addition, from the approximate positioning of the peaks, the nature of the water present in the material can be identified. For example, for the case of spruce wood, concerning the longitudinal relaxation time $T_{1}$, the bound water appears around $1 \mathrm{~ms}$, whereas the free water appears around $50 \mathrm{~ms}$. These relaxation times are not the same for all materials, since they depend greatly on the size of the pores. Moreover, the signal integral under each peak (the area under the peak), leads to the quantification of the concerned water type (bound or free water).

In fact, the solid protons (that correspond to the solid matrix) are identified by very short $\mathrm{T}_{2}$ transverse relaxation times, which are not detected with the experimental protocol used. 
Nevertheless, these protons can have high $\mathrm{T}_{1}$ relaxation times. As a result, it is necessary to verify that the $T_{1}$ longitudinal relaxation times of the peaks correspond to the $T_{2}$ crosssectional relaxation times of the bound water and the free water. Figure 6-b shows the NMR diagram of $\mathrm{T}_{2}$ transverse relaxation times, in which two peaks are distinguished. The existence of these two peaks in this diagram validates the presence of bound and free water identified in Figure 6-a.

Since the $T_{1}$ NMR measurements were made every hour until the sample reaches equilibrium, a comparative study can be made. For each measurement, the areas under the peaks that correspond to the bound and free water were calculated, in order to quantify both water types function of the time. The results are finally interpreted, making the link with the swelling of the wooden cell walls, which is a function of the bound water within the material (El Hachem et al., 2019).

\subsection{Experimental protocol for chemical constituents' evaluation using XRD}

The interest of this experiment is to identify the crystalline phase in the material, and to understand the various interactions between the chemical constituents of spruce wood and humidity. Therefore, three cylindrical samples of $20 \mathrm{~mm}$ diameter and $2 \mathrm{~mm}$ thickness were prepared (the circular plane corresponding to the tangential plane of wood), and each one of them was big enough to contain a complete reproduction cycle of wood, where early wood and latewood coexist. The samples were conditioned in a climatic chamber with controlled temperature and relative humidity. The temperature was always $25{ }^{\circ} \mathrm{C}$, and the relative humidity states tested along the sorption cycle were $10 \%, 60 \%, 72 \%, 78 \%$ and $87 \%$. These levels were chosen in order to have refine relative humidity states around the FSP, as observed by (El Hachem et al., 2017).

The used XRD device is Bruker D2 Phaser Bragg-Brentano $(\theta / 2 \theta)$ diffractometer at SATIE (Systèmes et Applications des Technologies de l'Information et de l'Energie) in ENS-ParisSaclay, France. The device in shown in Figure 7-a. It is equiped with an X-ray tube, provided with a cobalt anticathode $(\lambda=1.78897 \AA)$ and a LYNXEYE linear detector with a maximum opening of $10^{\circ}$, allowing a significant gain in intensity and a fast acquisition of diffraction diagrams. Slots of $0.6 \mathrm{~mm}$ and $8 \mathrm{~mm}$ were respectively adopted for the source and the detector. 
A sample holder with an X-ray transparent dome was used. The dome is tightened to the support through a waterproof rubber wall. An anti-diffusion knife was also used.

At each relative humidity, when a sample reached mass equilibrium, it was covered by the dome inside the climatic chamber, and then used to perform the corresponding XRD test. The used dome is shown in Figure 7-b. After the XRD test, the sample was placed in a climatic chamber programmed to a different relative humidity level.

The diagrams presented in this study were completed over an angular interval between $5^{\circ}$ and $70^{\circ}$. An acquisition time of $1 \mathrm{~s}$ per point and a pitch of $0.02^{\circ}$ were chosen. A high voltage of $30 \mathrm{kV}$ and a current of $10 \mathrm{~mA}$ were used.

For this study, the post-treatment was completed using Diffrac.EVA software. The purpose is to follow the evolution of the diffraction diagrams from one water state to another.

Therefore, crystalline and amorphous phases as well as instrumental background noise were identified. In the quantification method of the amorphous phase, the instrumental background noise was subtracted. Then the crystalline phase was recognized based on the PDF-2 sheets.

Indeed, in the diffraction diagram containing several phases, the peak intensity ratio of the various constituents depends on the ratio of their proportion. This is the basic principle of quantitative XRD methods. Since the notion of intensity does not mean the use of a diffraction peak height, as this can lead to errors that can reach $20 \%$ (Cyr et al., 1998), the diffraction peak intensity means the area between the curve and the continuous bottom line. This net surface is known by integral intensity. Therefore, qualitatively, in the case of a phase mixture, the greater the area of the peaks of a phase, more this phase is present in large proportion. It is subsequently possible to identify the composition of the material in weight proportions. In this work, since cellulose is the only chemical constituent present in the crystalline phase, any detected crystalline peak will be assimilated to it. Concerning the amorphous phase, it can be quantified by assuming that the intensity of the halo due to the scattered X-ray disorder is proportional to the surface under the proper hump (Cyr et al., 1998).

The surface method consists of calculating the amorphous phase ratio as the ratio of the halo surface to that of the entire diffraction diagram. The different surfaces are calculated using Diffrac.EVA software, after correcting the diffraction diagrams. 
The procedure consists therefore to first substract background noise from the diffraction diagrams. Then, the area of the amorphous phase is deduced by subtracting the area of the background noise diagram from the area under the curve of the halo. Finally, the crystalline phase is defined by subtracting the areas of background noise and amorphous phase from the total surface of the diffraction diagram.

Hence, the amorphous phase rate and the crystalline phase rate can be calculated. Each phase is then represented on a diffractogram. The crystalline phases are compared with those tabulated in PDF-2 sheets. The evolution of the crystalline and amorphous phases as a function of the relative humidity are studied. This is governed by the monitoring of the area under the curve which represents the amorphous phase, by the monitoring of the half-height width of the crystalline phase, and finally by the follow-up of the inter-reticular distances of the crystalline phase.

\section{Results and discussion}

Results of the detailed above experimental techniques and procedures will show first the follow up and quantification of water sorption, then the evolution of the cell walls chemical constituents along the sorption cycle, and will be followed by a global interpretation of those results.

\subsection{Follow up and quantification of water sorption by NMR}

The follow up of different water states (free, bound and constitutive) in the material in the sorption cycle was studied by NMR, and was achieved function of time, in order to study the evolution of the state of water. In fact, the dimensional variations of the cell walls caused by the environmental hygric variations are function of bound water presence within the material. This part of the study will lead to a better understanding of the water state, and a better evaluation of the dimensional variations function of the relative humidity.

The NMR diagrams corresponding to the longitudinal relaxation time $\mathrm{T}_{1}$, obtained from a dry state up to a relative humidity of $78 \%$ are shown in Figure 8 -a. Acquisitions were taken with a gap of one hour, except that in this figure an interval of 12 hours is retained for clarity purposes. For each acquisition, two peaks are discerned: the peak around $1 \mathrm{~ms}$ corresponds to the bound water, and the peak between 50 and $100 \mathrm{~ms}$ corresponds to the free water. 
From a general point of view, the peak representing the bound water evolves with time. As already mentioned, it is the area under the peaks that must be considered. The areas under each peak, calculated at each acquisition, are shown in Figure 8-b. This figure shows that the amount of bound water, initially zero in the material, increases rapidly over time, until it reaches a constant value. On the other hand, the free water remains more or less constant. This is justified by the fact that the saturation point of the fibers corresponds to a relative humidity greater than 78\% (El Hachem et al., 2017).

In a next conditioning step, the samples being conditioned at $78 \%$ relative humidity, a small tube containing a salt solution that guarantees a relative humidity of $95 \%$ was placed in the NMR tube. The acquisitions were triggered immediately. Figure 9-a shows the NMR diagrams corresponding to the longitudinal relaxation time $T_{1}$, obtained from a relative humidity of $78 \%$ to $95 \%$. As before, acquisitions are presented with an interval of 12 hours. The two peaks representing bound and free water are always present. However, in this case, both peaks evolve with time. The areas under each peak, calculated at each acquisition, are shown in Figure 9-b. This figure shows that the areas below the two peaks increase as a function of time. On the other hand, especially the first hours of the experience, the bound water kinetics is greater than that of free water. In fact, the saturation point of the fibers corresponds to a relative humidity between $78 \%$ and $95 \%$, due to which, up to the relative humidity of $95 \%$, the quantities of bound water and free water evolve at the same time within the material. In addition, since the saturation point of the fibers has not been reached at $78 \%$ relative humidity, the bound water is first absorbed faster than the free water.

\subsection{Evolution of the chemical constituents on the sorption cycle using XRD}

A study at the level of the chemical constituents of the cell walls was achieved. In fact, the different properties of these constituents are at the base of the physical phenomena that take place on the scale of the cell walls. Firstly, the complex structure of the cell walls consists of several layers and sub-layers, each having mainly specific proportions of cellulose, hemicellulose and lignin. The water absorption associated with this scale is therefore highly dependent on the physical parameters of the chemical constituents. As a result, the use of XRD technique helps to quantify and evaluate the evolution of the different phases proportions as a function of the relative humidity, which will also provide information about the reactions between chemical molecules and relative humidity. On the other hand, the distances between the crystalline molecules can be calculated according to the hydrous state 
of the material, giving more information about the local compressive or tensile stresses that may exist due to local dimensional variations of the cell walls (Yamamoto et al., 2010).

An example of a diffraction diagram obtained is shown in Figure 10. A first estimate of the background noise and the amorphous phase was first performed, in order to identify the crystalline phase. Then, the half-height width of the crystalline peak of the [002] plane and the corresponding inter-reticular distance were calculated. This approach was applied to each hygric state.

The curves representing the background noise and the amorphous phase for each treated water condition are shown in Figure 11-a. These results showed that up to a relative humidity of $78 \%$, the amount of amorphous phase decreases as the relative humidity increases. On the other hand, between relative humidity of $78 \%$ and $87 \%$, the amount of amorphous phase increases.

Several elements should be considered for the results' analysis. Actually, the bound water is absorbed by the cell walls, and particularly by the amorphous phase of the walls. This takes place through the breaking of hydrogen bridges in hemicellulose and amorphous cellulose. As relative humidity increases, there is a possibility that hemicellulose and amorphous cellulose in the cell walls dissolve (Kulasinski et al., 2015), causing a decrease in the amorphous phase (Figure 11-a).

As the FSP corresponds to a relative humidity between $78 \%$ and $95 \%$, free water takes place within the material, as demonstrated in section 3.1, but appears in XRD diagrams as amorphous phase (Brownstein et al., 1979; Borgia et al., 2000; Keeler, 2011). The increase of the amorphous phase between $78 \%$ and $87 \%$ relative humidity is therefore due to the presence of free water within the material.

The curves representing the crystalline phase below the peak [002] for each treated relative humidity condition are shown in Figure 11-b. The changes in inter-reticular distances function of the relative humidity are shown in Figure 12. In addition, the areas under the curves and the widths at half height of the peaks show no significant variation from a relative humidity to another. Figure 12 further shows that the inter-reticular distances decrease when the relative humidity increases. 
Indeed, the crystalline phase, representing only the crystalline cellulose, is hydrophobic (Kulasinski et al., 2015, Park et al., 2010). It is well oriented and organized to prevent interactions with moisture. Its amount remains therefore more or less constant. This is proved by verifying the width at half height of the crystalline peak [002] and by the area under the curve. On the other hand, the reduction of inter-reticular distances from one hygric state to another can be caused by mechanical stresses of compression in the secondary layer of the cell walls due to swelling. In fact, swelling dimensional changes of hemicellulose and amorphous cellulose generate compressive stresses at the level of the crystalline cellulose in the secondary layer. This can be the cause of the inter-reticular distances decrease in the cellulose mesh.

\subsection{Global interpretation}

It has been shown, by the use of the NMR technique, that below a relative humidity of $78 \%$, there was only bound water within the material and the FSP was not yet reached. On the other hand, between relative humidity of $78 \%$ and $95 \%$, the bound and the free water coexisted within the material. The FSP therefore corresponds to a water content that has been reached between these two levels of humidity. Hence, it is consistent that above $78 \%$ relative humidity, the X-ray micro-tomography results obtained in the previously published paper (El Hachem et al., 2017) were influenced by the presence of free water in the porosity.

On the other hand, the XRD reported constructive explanations regarding the shrinkage of cell walls observed below the FSP. Indeed, when absorbing moisture, a certain proportion of the hemicelluloses may dissolve due to hydrogen bridges rupture, or may swell and tend to generate compressive stresses at the level of the secondary wall, and more particularly on the crystalline cellulose. These stresses cause a decrease in the inter-reticular distances of the cellulose molecules. These phenomena can generate the local swelling along the longitudinal axis of the wood cell walls and the local shrinkage in the transversal plane of the cell walls.

It is after all important to evaluate the coupling of the previous results, concerning the bound and free water uptake within the material, the chemical components evolution due to the increase of the relative humidity, and the previous results obtained by (El Hachem et al., 2017) about the microscopic morphological changes along the sorption cycle, many interesting. The results elaborated in this paper are totally consistent with previously 
described results based on X-ray micro-tomography experimental study (El Hachem et al., 2017).

Therefore, it is to be noted that below the FSP, when the relative humidity increases, spruce wood is subjected to a macroscopic swelling, whereas, at the microscopic scale, the cell walls absorb bound water through the hemicellulose, which leads to local swelling of the cell walls along the longitudinal axis and to a stretching of the cell walls in the transversal plane. This leads to a shrinkage of the walls in that plane (the thickness of the cell walls decreases), and to an increase of the pore diameter. On the other hand, after reaching the FSP, there is no more obvious macroscopic swelling, whereas, at the microscopic scale, the cell walls are already saturated in bound water, and the free water takes place in the porosity holes.

\section{Conclusion}

Two experimental campaigns have been conducted in this study for a better characterization of the hygro-mechanical interactions within the wood anatomical structure. At a first stage, NMR was investigated to evaluate and quantify the increase in bound and free water within the material. This study was done along the sorption cycle and for different relative humidity levels. XRD was then performed for a better understanding of the interactions of moisture with the chemical constituents of wood cell walls.

The coupling of these two experimental campaigns, in addition to the works elaborated by (El Hachem et al., 2017) has led to interesting results. The results showed an increase of bound water within the material between dry state and the FSP. Once the FSP is reached, the cell walls are already saturated in bound water, and the increase in relative humidity induces an increase in free water within the material. On the other hand, below the FSP, hemicellulose absorbs moisture and swells. In addition, a certain proportion of hemicelluloses may dissolve following the rupture of the hydrogen bridges. As for the amount of crystalline cellulose, it did not vary according to the relative humidity. A small narrowing of the crystalline cellulose molecules was observed, due to compressive stresses exerted by the swelling of the hemicellulose. These results are in agreement and even justify the results obtained with (El Hachem et al., 2017). 
These results also provide insight into the interaction of relative humidity and wood with more precision on the nature of water in the wood cells and its dimensional variations during swelling. These interactions should be considered seriously when modeling the multiphysical transfers phenomena inside this material.

\section{References}

Almeida, G., Leclerc, S., and Perre, P. (2008). NMR imaging of fluid pathways during drainage of softwood in a pressure membrane chamber. Int. J. Multiph. Flow 34, 312-321.

Almeida, G., Huber, F., Perré, P. (2014). Free shrinkage of wood determined at the cellular level using an environmental scanning electron microscope. Maderas Cienc. Tecnol. 16, 187198.

Arends, T., Barakat, A.J., Pel, L. (2018). Moisture transport in pine wood during one-sided heating studied by NMR. Experimental Thermal and Fluid Science 99, 259:271.

Bonnet, M., Courtier-Murias, D., Faure, P., Rodts, S., Caré, S. (2017). NMR determination of sorption isotherms in earlywood and latewood of Douglas fir. Identification of bound water components related to their local environment. Holzforschung 71, 481-490.

Borgia, G.C., Brown, R.J.S., and Fantazzini, P. (2000). Uniform-penalty inversion of multiexponential decay data: II. Data spacing, T2 data, systematic data errors, and diagnostics. J. Magn. Reson. 147, 273-285.

Brownstein, K.R., and Tarr, C.E. (1979). Importance of classical diffusion in NMR studies of water in biological cells. Phys. Rev. A 19, 2446-2453.

Cueff, G., Mindeguia, J.C., Dréan, V., Breysse, D., Auguin, G. (2018). Experimental and numerical study of the thermomechanical behaviour of wood-based panels exposed to fire. Construction and Building Materials 160, 668-678.

Cyr, M., Husson, B., and Carles-Gibergue, A. (1998). Détermination, par diffraction des rayons $\mathrm{X}$, de la teneur en phase amorphe de certains matériaux minéraux. J. Phys. IV 08, Pr523-Pr5-30.

Derome, D., Rafsanjani, A., Hering, S., Dressler, M., Patera, A., Lanvermann, C., SedighiGilani, M., Wittel, F.K., Niemz, P., Carmeliet, J. (2013). The role of water in the behavior of wood. J. Build. Phys. 36, 398-421.

Dodoo, A., Gustavsson, L., and Sathre, R. (2012). Effect of thermal mass on life cycle primary energy balances of a concrete- and a wood-frame building. Appl. Energy 92, 462472.

Eitelberger, J., Hofstetter, K. (2011). A comprehensive model for transient moisture transport in wood below the fiber saturation point: physical background, implementation and experimental validation. International Journal of Thermal Sciences 50, 1861-1866.

El Hachem, C., Abahri, K., Bennacer, R. (2019). Original experimental and numerical approach for prediction of the microscopic hygro-mechanical behavior of spruce wood. Construction and Building Materials 203, 258-266. 
El Hachem, C., Abahri, K., Vicente, J., Bennacer, R., Belarbi, R. (2018). Hygromorphic characterization of softwood under high resolution X-ray tomography for hygrothermal simulation. Heat and Mass Transfer, https://doi.org/10.1007/s00231-018-2311-9.

El Hachem, C., Abahri, K., Bennacer, R. (2017). Microscopic swelling analysis of spruce wood in sorption cycle. Energy Procedia 139, 322-237.

Gezici-Koç, Ö., Erich, S.J.F., Huinink, H.P., Van der Ven, L.G.J., Adan, O.C.G. (2017). Bound and free water distribution in wood during water uptake and drying as measured by 1D magnetic resonance imaging. Cellulose 24, 535-553.

Gezici-Koç, Ö., Erich, S.J.F., Huinink, H.P., Van der Ven, L.G.J., Adan, O.C.G. (2018). Understanding the influence of wood as a substrate on the permeability of coatings by NMR imaging and wet-cup. Progress in Organic Coatings 114, 135-144.

Guo, J., Song, K., Salmén, L., Yin, Y. (2015). Changes of wood cell walls in response to hygro-mechanical steam treatment. Carbohydr. Polym. 115, 207-214.

Hernández, R.E., and Cáceres, C.B. (2010). Magnetic resonance microimaging of liquid water distribution in sugar maple wood below fiber saturation point. Wood Fiber Sci. 42, 259-272.

Ju, X., Bowden, M., Brown, E.E., and Zhang, X. (2015). An improved X-ray diffraction method for cellulose crystallinity measurement. Carbohydr. Polym. 123, 476-481.

Keeler, J. (2011). Understanding NMR spectroscopy (John Wiley \& Sons).

Kulasinski, K., Guyer, R., Derome, D., and Carmeliet, J. (2015). Water adsorption in wood microfibril-hemicellulose system: role of the crystalline-amorphous interface. Biomacromolecules 16, 2972-2978.

Langford, J.I., and Louër, D. (1996). Powder diffraction. Rep. Prog. Phys. 59, 131.

Liang, T., Meihua, W., Sufen, H., and Mingshu, T. (1997). Method to evaluate the reactivity of microcrystalline quartz in carbonate rock. Adv. Cem. Res. 9, 55-63.

Mäkinen, H., Jaakkola, T., Piispanen, R., Saranpää, P. (2007). Predicting wood and tracheid properties of Norway spruce. Forest Ecology and Management 241, 175-188.

Neagu, R.C., and Gamstedt, E.K. (2007). Modelling of effects of ultrastructural morphology on the hygroelastic properties of wood fibres. J. Mater. Sci. 42, 10254-10274.

Park, S., Baker, J.O., Himmel, M.E., Parilla, P.A., and Johnson, D.K. (2010). Cellulose crystallinity index: measurement techniques and their impact on interpreting cellulase performance. Biotechnol. Biofuels 3, 10.

Rafsanjani, A. Lanvermann, C. Niemz, P. Carmeliet, J. Derome, D. (2013). Multiscale analysis of free swelling of Norway spruce. Composites Part A 54, 70-78.

Rostom, L., Courtier-Murias, D., Rodts, S., Caré, S. (2019). Investigation of the effect of aging on wood hygroscopicity by 2D 1 H NMR relaxometry. Holzforschung, https://doi.org/10.1515/hf-2019-0052. 
Salvati, E., Brandt, L.R., Uzun, F., Zhang, H., Papadaki, C., Korsunsky, A.M. (2018). Multiscale analysis of bamboo deformation mechanisms following $\mathrm{NaOH}$ treatment using $\mathrm{X}$ ray and correlative microscopy. Acta Biomaterialia 72, 329-341.

Wang, M., and Pan, N. (2008). Predictions of effective physical properties of complex multiphase materials. Mater. Sci. Eng. R 63, 1-30.

Wang, W., Huang, Y., Cao, J., Zhu, Y. (2018). Penetration and distribution of paraffin wax in wood of loblolly pine and Scots pine studied by time domain NMR spectroscopy. Holzforschung 72, 125-132.

Yamamoto, H., Ruelle, J., Arakawa, Y., Yoshida, M., Clair, B., and Gril, J. (2010). Origin of the characteristic hygro-mechanical properties of the gelatinous layer in tension wood from Kunugi oak (Quercus acutissima). Wood Sci. Technol. 44, 149-163.

Zhou, M., Caré, S., Courtier-Murias, D., Faure, P., Rodts, S., Coussot, P. (2018). Magnetic resonance imaging evidences of the impact of water sorption on hardwood capillary imbibition dynamics. Wood Science and Technology 52, 929-955.

Zhou, M., Caré, S., Kind, A., Courtier-Murias, D., Rodts, S., Gerber, G., Aimedieu, P., Bonnet, M., Bornert, M., Coussot, P. (2019). Wetting enhanced by water adsorption in hygroscopic plantlike materials. Physical Review Research 1, 033190.

Zlahtic-Zupanc, M., Lesar, B., Humar, M. (2018). Changes in moisture performance of wood after weathering. Construction and Building Materials 193, 529-538. 